Cahiers de recherche sociologique

RECHERCHE

SOCIOLOGIQUE

\title{
Pensée psychotique, technologies des communications et réadaptation psychosociale
}

\section{Jean-François Pelletier}

Numéro 41-42, 2005

Nouveau malaise dans la civilisation : regards sociologiques sur la santé mentale, la souffrance psychique et la psychologisation

URI : https://id.erudit.org/iderudit/1002469ar

DOI : https://doi.org/10.7202/1002469ar

Aller au sommaire du numéro

\section{Éditeur(s)}

Département de sociologie - Université du Québec à Montréal

\section{ISSN}

0831-1048 (imprimé)

1923-5771 (numérique)

Découvrir la revue

Citer cet article

Pelletier, J.-F. (2005). Pensée psychotique, technologies des communications et réadaptation psychosociale. Cahiers de recherche sociologique, (41-42), 257-278. https://doi.org/10.7202/1002469ar
Résumé de l'article

Cet article propose une réactualisation de la théorie de la communication paradoxale (école de Palo Alto) à la lumière des technologies de l'information et des communications, très en vogue de nos jours. Après avoir campé les grands enjeux théoriques de cette approche antipsychiatrique, cet exposé revient sur diverses modalités d'exercice de la communication comme moyen de favoriser la réadaptation psychosociale des personnes ayant, ou ayant eu des problèmes de santé mentale. Internet émerge alors comme un secteur à explorer et à développer. D'une part, son caractère multimédia en fait un mode de communication typiquement paradoxal. D'autre part, il fait en sorte que soient cumulés les avantages des diverses techniques étudiées (art-thérapie, écriture journalistique, radio et télévision), ce qui facilite l'expression d'un discours affranchi de l'ordre raréfiant du discours institutionnel. Ce dernier dénigre en effet la parole plus ou moins chaotique du patient en en faisant le symptôme d'une maladie à juguler. C'est ainsi que, grâce au potentiel thérapeutique d'Internet, la pensée psychotique peut recouvrer une certaine valeur et c'est ainsi que ses porteurs peuvent communiquer avec autrui, c'est-à-dire directement avec la société et aussi avec un certain au-delà transcendantal. 


\section{Pensée psychotique, technologies des communications et réadaptation psychosociale}

Jean-François PELLETIER

Cet article propose de situer les technologies de l'information et des communications (TIC) en contexte de réadaptation psychosociale, et ce, en continuité avec la théorie de la communication qui tente d'expliquer la schizophrénie autrement qu'en en faisant un dysfonctionnement neurobiologique (école de Palo Alto). Un bref rappel des grandes lignes de cette théorie, généralement associée à l'antipsychiatrie, sera présenté en guise d'introduction. Ensuite, sur un terrain moins théorique et plus technique, l'emploi des TIC pour fin de réadaptation sera comparé à d'autres approches utilisant la communication comme moyen de réinsertion (artthérapie, radio et télévision). Finalement, revenant sur des considérations davantage philosophiques, nous proposerons une discussion dans le cadre de laquelle la pensée psychotique sera mise à profit, voire réhabilitée, notamment avec l'émergence de ce que Hervé Fischer appelle la lecture kangourou, issue de la révolution numérique ${ }^{1}$.

Nous verrons que la réalisation d'un projet qui passe par les TIC peut mettre à contribution l'originalité de la pensée psychotique. Redevenant une rareté recherchée, cette dernière pourra ainsi recouvrer une certaine valeur, et ce, au grand soulagement de ceux qui la portent. En effet, toujours selon Hervé Fischer, les technologies et la révolution numériques sonnent le glas de l'autoritarisme didactique et ébranlent l'ordre classique des discours. Elles pavent ainsi la voie à l'émergence de possibilités nouvelles quant à la réhabilitation de certains de nos concitoyens jusqu'à maintenant déconsidérés justement parce que leur pensée échappe à la linéarité logico-déductive (ou est-ce l'inverse?), étant qualifiée d'irrationnelle et classée comme pathologique.

1. H. Fischer, «Les machines éducatives et les hommes». Dîner-conférence du colloque Les TIC: valeur ajoutée à la formation, Université du Québec à Trois-Rivières, 2003. 
Pour situer la théorie de la communication, rappelons d'abord qu'il n'existe toujours pas de test, sanguin ou autre, qui permette d'établir objectivement un diagnostic de maladie mentale. Pour établir un tel diagnostic, il faut interpréter une conduite comme symptomatique d'une pathologie (acting out). On ne peut encore que recourir à l'observation, directe ou indirecte, des comportements d'un individu et lorsque tel ou tel de ses comportements attire l'attention, on l'interprète puis on lui donne éventuellement un nom. C'est donc une question d'interprétation et, à ce titre, l'appréciation ne peut être au moins que partiellement subjective.

En Occident, le législateur a déterminé que ce n'est qu'un médecin psychiatre qui peut établir un diagnostic de maladie mentale, avec toutes les conséquences qui peuvent en découler (privation de liberté et internement, prise quotidienne de médicaments, effets secondaires de ceux-ci, stigmatisation, etc.). Cette étiquette permet ensuite la classification qui préfigure le type d'intervention à appliquer au comportement problématique ou le genre de médicament(s) à prescrire. Ainsi, l'esprit de classification qui caractérise tant le modèle médical se présente sous la forme d'une nosographie des maladies mentales et de leurs traitements. Elle prend la forme, par exemple, d'un manuel bien connu: le Diagnostic and Statistical Manual of Mental Disorders (DSM).

Or selon Michel Foucault ${ }^{2}$, tout discours, toute discipline discursive est un mécanisme de raréfaction, de sorte qu'on ne peut parler en expert que si on a été formé par les professionnels de la profession, laquelle se dit de tel ou tel discours: médical, juridique, théologique, etc. C'est donc le Collège des médecins qui valide et confirme que telle ou telle pratique est ou n'est pas de la médecine. Toutefois, le fait d'associer la santé mentale à la médecine ne fait heureusement plus l'unanimité. Avec l'avènement, dans les années 1960, des contestations diverses et de l'antipsychiatrie en particulier, on a voulu rendre la parole à la personne qui vit le problème. Il s'agissait de conférer à cette parole autant d'importance qu'on en accorde au savoir psychiatrique lorsque vient le temps d'expliquer une situation problématique et de lui trouver des pistes de solution.

$\mathrm{La}$ «folie» peut-elle parler en son propre nom? La parole du «malade mental», parfois exprimée sous la forme du récit de ses délires, peut-elle avoir statut de discours? Si oui, de quelle façon ce discours peut-il faire son chemin jusqu'à des interlocuteurs pour qu'éventuellement ces derniers puissent contribuer à la compréhension des mécanismes traditionnels de

2. M. Foucault, L'ordre du discours, Paris, Gallimard, 1971. 
l'exclusion sociale et à leur déconstruction? C'est en ces termes que cet article se propose de réactualiser la théorie antipsychiatrique de la communication, qui est fait la théorie de la communication paradoxale.

\section{La communication paradoxale}

Le double langage est une communication considérée comme paradoxale parce qu'il se déploie à au moins deux niveaux, lesquels se contredisent souvent. Nombreux sont ceux qui ne peuvent le soutenir ou qui y voient mensonge et injustice. En effet, des sociologues comme Erving Goffman ont montré que le discours, c'est-à-dire le véritable sens d'un message, est aussi émis, peut-être davantage que par la parole, par un langage non verbal ${ }^{3}$. L'analyse du langage non verbal ne doit d'ailleurs pas se restreindre aux seuls mouvements corporels connus sous le nom de kinesthésie. Selon les précurseurs de la théorie de la communication, il faut considérer posture, gestuelle, mimique, inflexions de la voix, rythme et intonation des mots, «et toute autre manifestation non verbale dont est susceptible l'organisme, ainsi que les indices ayant valeur de communication ${ }^{4} »$. Ce sont ces épiphénomènes, parfois difficiles à cerner, qui meublent le théâtre de l'interaction ${ }^{5}$, et c'est là que le véritable partage des pouvoirs se joue ou ne se joue pas. Dans le feu de l'interaction, thérapeutique notamment, la domination de l'un peut très bien s'exercer sans que rien ne se dise et on suppose que la compréhension de ce phénomène devrait permettre de le neutraliser. D'où l'importance de le faire connaître du plus grand nombre, soit du public en général et pas seulement des spécialistes impliqués ou de l'entourage immédiat d'un cas particulier.

C'est en 1950 que le groupe de chercheurs, connu comme l'école de Palo Alto, a commencé à étudier le mode de communication pathologique qui existait dans des familles de schizophrènes. En 1956, ils ont publié le résultat de leur étude à l'effet que le paradoxe soit un modèle de communication qui mène à la double contrainte (double langage n'offrant aucune issue). Le paradoxe est un message qui, en même temps, nie ce qu'il affirme et affirme ce qu'il nie. Par exemple, l'injonction "sois spontané» est un paradoxe. On ne peut être spontané sur commande, et le destinataire d'un tel message est coincé. La double contrainte est une injonction

3. E. Goffman, Asiles: études sur la condition sociale des malades mentaux et autres reclus, Paris, Les Éditions de Minuit, 1968.

4. P. Watzlawick et al., Une logique de la communication, Paris, Seuil, 1972.

5. Y. Winkin, Les moments et leurs hommes, Paris, Seuil-Minuit, 1988. 
paradoxale tant au niveau cognitif qu'affectif, car aucune réponse n'est possible. L'image classique utilisée pour illustrer ce phénomène est celui d'une mère qui, à la rencontre de son enfant, lui reproche de ne pas chercher à l'embrasser («Tu ne m'embrasses pas?»), alors qu'elle-même reste affectivement très distante en maintenant une position corporelle de fermeture ou en repoussant son enfant aussitôt que possible après qu'il l'eut embrassée. L'enfant ne sachant à quel message répondre, il devient confus et hésitant. Ce pattern se répétant de manière récurrente, la confusion et l'hésitation deviendront elles aussi récurrentes avec le développement de la personnalité schizoïde de l'enfant. Dans ce type de relation, il est en effet impossible de faire le bon choix, à moins de la dénoncer. Le destinataire du message paradoxal est mis dans l'impossibilité de sortir du cadre fixé par ce message, qu'il ne peut critiquer ou éviter, sous peine de passer pour l'instigateur d'un conflit. Or, si logiquement le message est dénué de sens, il possède néanmoins une réalité pragmatique qui fait qu'on «ne peut pas ne pas y répondre, mais on ne peut pas non plus y réagir de manière adéquate (c'est-à-dire non paradoxale) puisque le message lui-même est paradoxal ${ }^{6}$ ». D'où l'incongruité, l'étrangeté d'une conduite réellement paradoxale, la bizarrerie qui fait qu'on parle de déraison et que l'on considère que l'acting out étant la manifestation observable d'une maladie mentale. C'est ainsi que la théorie de la communication explique ce que nous connaissons sous le nom de schizophrénie.

Gregory Bateson et l'école de Palo Alto précisent donc que la famille est le premier agent d'exercice de cette communication paradoxale. Cependant leur modèle déçoit parce qu'ils annoncent une dimension clinique qu'ils ne développent pas réellement. "Communication analysis has championed the existence of innate processes which influence communication, but it is limited by the lack of empirical research ${ }^{7} . »$ Watzlawick et compagnie, disciples de Bateson, avouent le caractère approximatif de leurs propositions. Il leur semble en effet que c'est au niveau de l'expérience «mystique» que se produit le changement thérapeutique, «changement dont, après une thérapie réussie, on ne peut dire ni comment ni pourquoi, il s'est produit, et en quoi finalement il consiste ${ }^{8}{ }^{\prime}$. Or, dans le

6. P. Watzlawick, op. cit.

7. E. Bradley et H.-J. Biedermann, «Bandler and Grinder's Neurolinguistic Programming: its Historical Context and Contribution», Psychotherapy, vol. 22, $\mathrm{n}^{\circ} 1$, printemps 1985 .

8. P. Watzlawick, op. cit. 
DSM et la littérature connexe, la subjectivité, soit l'interprétation que le patient peut formuler à propos de son problème, est le mal par lequel se développent parfois les désordres religieux (religious desorders). En effet, le patient parle souvent de son problème comme relevant de la spiritualité: «emanating from the narratives is the important role played by religious signifiers ${ }^{9} \gg$. Il semble avoir conscience que sa parole, et par extension luimême, n'a que peu de valeur. Pour plusieurs, elle n'a pas de sens, elle est insensée et insignifiante ${ }^{10}$. Ainsi, c'est la vie du patient qui manque de sens et c'est ce qu'il voudrait bien retrouver.

De nombreux témoignages de patients psychiatriques, dont celui de Martin Fortier sur lequel nous reviendrons dans la section suivante, font état de la présence d'une communication de type paradoxale dans le giron familial. Cette présence est souvent décrite comme le mécanisme premier par lequel un individu se retrouve marginalisé. Cette mise à l'écart plus ou moins intentionnelle a des conséquences très importantes parce qu'elle se fait par rapport à des proches dont on attend des manifestations d'affection plutôt qu'une attitude générant cette mise à l'écart. Cette situation, lorsque persistante, mine la personnalité en développement d'un individu, lequel perd ses repères et ne parvient pas à se situer autrement qu'en marge, d'abord du noyau familial, puis de la société. La schizophrénie se définit alors comme une stratégie d'évitement d'une relation basée sur la communication paradoxale (plutôt que seulement consécutive à un dérèglement neurochimique), et dès lors que cette stratégie a porté quelque fruit (pouvoir se retirer dans sa chambre lors d'événements sociaux par exemple), il devient difficile, très difficile de s'en défaire. C'est pourquoi les thérapies qui s'inspirent de l'école de Palo Alto visent à faire prendre conscience à un individu de la présence de la communication paradoxale dont il a été victime et de ses conséquences. Malheureusement, il est souvent trop tard parce que cet individu n'a pas développé les habiletés nécessaires à distinguer sens propre et sens figuré. Les intervenants qui accompagnent ce type de personnes savent qu'elles ont parfois tendance à tout prendre au pied de la lettre et qu'il faut être prudent, même lorsqu'il s'agit simplement de se montrer chaleureux ou de faire de l'humour (paranoïa).

9. E. Corin, «The thickness of being: intentional worlds, strategies of identity, and experience among schizophrenics», Psychiatry: Interpersonal and Biological Processes, vol. 6, été 1998, p. 133-146.

10. R. M. Bergner, "Therapeutic approaches to problems of meaninglessness», American Journal of Psychotherapy, vol. 52, hiver 1998, p. 72-87. 
Il n'est donc pas toujours simple d'expliquer à une personne à tendance schizoïde en quoi consiste le phénomène de la communication paradoxale dont elle a pu être victime. De même, mais pour d'autres raisons, les membres de sa famille ne se montrent pas toujours ouverts à une théorie qui les désignent au moins en partie responsables de la situation.

La présence d'une communication de type paradoxal, verbale ou non verbale, peut être plus facile à admettre de la part d'un observateur extérieur à cette communication. En effet, si on étudie isolément un individu qui manifeste des comportements considérés comme psychopathologiques, la recherche portera sur la nature de cet état. Mais si on inclut dans cette recherche les effets du comportement sur autrui, de même que les réactions d'autrui à ce comportement et le contexte où tout cela se déroule, alors «l'accent se déplace de la monade artificiellement isolée à la relation qui existe entre les différentes parties d'un système plus vaste ${ }^{11}$ ». C'est pourquoi la nature de la relation, qui dépend de «la ponctuation des séquences de communication entre les partenaires ${ }^{12} »$, devient plus facilement visible aux yeux d'un tiers. Cooper et Laing considèrent eux aussi ce tiers pris à témoin comme indispensable, car c'est lui «qui unifie la dyade à travers la médiation ${ }^{13}$ \%. C'est lui qui peut voir que les comportements sont liés, dès lors qu'ils sont remis dans leur perspective. Sans lui, la communication demeure incompréhensible. C'est donc la présence $\mathrm{du}$ tiers et la triangulation qu'elle permet qui fait que la domination verbale/non verbale devient patente et non plus seulement que latente, et c'est à partir de ce moment, en théorie, qu'on peut s'en libérer.

Nous allons maintenant esquisser quelques expériences communautaires qui ont cherché à favoriser le rétablissement de personnes en processus de réadaptation psychosociale par leur appropriation de divers moyens de communication. Nous reviendrons ensuite sur l'utilité transcendantale du tiers.

11. P. Watzlawick, op. cit.

12. Id.

13. D. Cooper et R. Laing, Raison et violence: dix ans de la philosophie de Jean-Paul Sartre, Paris, Petite Bibliothèque Payot, 1971. 


\section{L'art-thérapie}

L'art-thérapie vise l'expressivité des émotions d'un individu en processus de réadaptation par le biais de l'apprentissage et de l'exercice d'une pratique artistique, notamment le dessin et la peinture. Elle vise aussi le développement d'une certaine discipline de travail et le recouvrement d'habiletés sociales par la fréquentation d'un atelier par exemple, mais son objectif principal est de permettre la libération des sentiments refoulés plus ou moins profondément dans le psychisme de l'individu. La présence de ces sentiments complexes limite la personne dans sa capacité à spontanément en vivre d'autres, de manière plus fluide. Il en résulte un inconfort et une difficulté à entrer socialement en contact avec autrui. Parmi ces sentiments, il y a celui qui découle du fait d'avoir été victime d'ostracisme (colère, déception, etc.).

La mission de la Fondation pour l'art thérapeutique et l'art brut du Québec, connue sous le nom de Les Impatients, est d'offrir un lieu d'expression artistique aux personnes ayant ou ayant eu des problèmes de santé mentale. Les ateliers d'art-thérapie favorisent l'échange des Impatients avec leurs proches et la communauté par la mise en valeur de leurs réalisations. Des rencontres individuelles sont également offertes au besoin, dans le but de faire un retour sur les images produites en atelier ${ }^{14}$. Bien connu du réseau montréalais de la santé mentale, Martin Fortier est un artiste complexe, membre des Impatients. L'analyse de quelques-unes de ses œuvres peut en révéler un riche contenu, qui a pour nous valeur de discours, comme nous allons maintenant le voir.

Dans une première œuvre ${ }^{15}$, Martin Fortier se représente à travers les traits d'un personnage en désarroi. Ses yeux sont rouges, et le fait que son regard soit fuyant symbolise le refus d'envisager une réalité qui lui déplaît. Collé sur la pupille de l'œil droit, on sent au toucher un grain qui confère du relief à cette partie du corps. Cette proéminence rappelle que le regard, bien que détourné, n'en est pas moins perçant. On voit également de très longs bras. Cette posture représente l'apitoiement, le désillusionnement. À propos des autres éléments conférant du relief à l'œuvre, Martin Fortier dit qu'ils rendent l'angoisse palpable. On voit également que le personnage est vêtu d'un pyjama. Il s'agit en fait d'une jaquette d'hôpital. On remarque de plus, vers le bas, des sphères qui sont les orbites oculaires des regards examinateurs et indiscrets croisés à l'urgence psychiatrique. Se sentant

14. http://www.impatients.ca.

15. http://polaristo.com/jfpelletier/cahiersderecherchesociologique/figure_1.gif. 
observé, le personnage se sent également jugé. En haut à gauche, un cercle clair constitue une lune. Davantage qu'une source de lumière et d'espoir, c'est plutôt le thème de la nuit qu'il figure.

Sur un autre tableau ${ }^{16}$, on voit aussi une lune se dessiner. Le regard comporte encore une fois du rouge, tandis que la bouche laisse voir des dents bien coupantes mais tachées. On pense ici aux soins corporels de base, comme l'hygiène buccale, qui sont souvent négligés lorsque la maladie sévit. Les couleurs rouge et orange forment le symbole religieux d'une auréole. Autour du visage, elles sont celles du feu, de la brûlure qui fait songer aux bûchers de l'Inquisition. À droite du personnage, on aperçoit des éléments de décors; une table avec, dessus, un calice. L'expérience de l'exclusion et de la psychose sont intimement liées aux préoccupations d'ordre spirituel et religieux, tel que nous l'avons mentionné plus haut. D'ailleurs une autre réalisation ${ }^{17}$ illustre principalement un calice. Martin Fortier sourit de prononcer ce mot qui, dans un autre contexte, pourrait être considéré comme un juron. «C'est une légende, j'étais sur l'Imipramine, un antipsychotique à déconseiller pour quelqu'un qui est bipolaire. J'hallucinais, puis c'est ça que je voyais quand je faisais mes tableaux ${ }^{18}$.» Un autre tableau ${ }^{19}$, où le personnage est encore une fois auréolé «prouve que j'étais parfaitement en santé. J'avais de l'angoisse, c'est déchiré, c'est quelqu'un qui est en train de se faire pendre, il crie au meurtre, il y a un serpent, il y a des visages, il pleure, il a des poissons qui sortent de la bouche $^{20} .{ }^{\prime}$ D'une autre image ${ }^{21}$, Martin Fortier en dit qu'il s'agit de sa «psychiatre dans sa bulle, dans une coupole transparente, comme une sainte $^{22} \gg$. Un dernier tableau ${ }^{23}$ représente encore une fois cette psychiatre. Toujours auréolée, elle joue dans le cœur de son patient. Celui-ci verse une larme parce que, selon l'auteur, sa jeunesse lui a été retirée le jour où on a posé sur lui un diagnostic psychiatrique.

Dans plusieurs des tableaux de Martin Fortier, on peut constater le proche voisinage qui met en relation le psychédélisme, le vécu psychiatrique et le mysticisme. Le psychédélisme est présent parce que Martin

16. http://polaristo.com/jfpelletier/cahiersderecherchesociologique/figure_2.gif.

17. http://polaristo.com/jfpelletier/cahiersderecherchesociologique/figure_3.gif.

18. M. Fortier, Entrevues, Folie Douce, Télévision communautaire de Vidéotron, hiver 1996.

19. http://polaristo.com/jfpelletier/cahiersderecherchesociologique/figure_4.gif.

20. M. Fortier, op. cit.

21. http://polaristo.com/jfpelletier/cahiersderecherchesociologique/figure_5.gif.

22. M. Fortier, op. cit.

23. http://polaristo.com/jfpelletier/cahiersderecherchesociologique/figure_6.gif. 
Fortier dit de l'un de ses tableaux qu'il s'agit de la transposition d'une hallucination. Dans un autre, l'éclatement correspond à sa propre psychose puisqu'il établit un lien entre le tableau et son état de santé, à propos duquel il fait de l'ironie. Le vécu psychiatrique et sa relation avec sa psychiatre sont le thème de deux des figures commentées, alors que ce personnage est présenté comme une «sainte». Le calice de l'une des réalisations, près duquel on retrouve un personnage vêtu d'une jaquette d'hôpital vu dans un autre tableau, témoigne lui aussi d'une préoccupation pour la spiritualité, ou plus spécifiquement pour le rituel religieux.

Dans l'œuvre peinte de Martin Fortier, force est donc de constater la grande place laissée à des thèmes religieux, à celui de l'angoisse et à la relation patient/psychiatre. Malgré que nombre de ses réalisations aient été entreprises lors de phases proches de la psychose, il se dégage une certaine cohérence d'un tableau à l'autre. Certes, sa pratique est davantage intuitive que déductive, puisqu'il s'agit d'une effusion créatrice relativement débridée, concomitante à des épisodes de psychose et d'hallucinations. Toutefois, l'artiste apparaît, dans cette optique, moins "naïf» qu'on pourrait d'abord le croire. Un certain nombre d'analyses et de récurrences sont présentes dans l'œuvre de Martin Fortier, laquelle peut ainsi être considérée comme un discours, véhiculant une certaine connaissance qui peut toucher le public et contribuer à la formation de futurs intervenants. Cependant, sans la présence de l'artiste pour commenter ses réalisations, leur compréhension reste limitée à un nombre restreint d'initiés, tandis que ces œuvres ne sont accessibles individuellement ou par petits groupes que lorsqu'elles sont exposées.

\section{La radio}

Le 10 octobre 1991, la première émission Folie Douce fut diffusée sur les ondes de Radio Centre-Ville à Montréal. Toujours en ondes en 2005, elle repose sur la participation de ceux qui connaissent bien les multiples facettes de la «folie», pour être des usagers du réseau de la santé mentale. $\mathrm{Au}$ début, les participants étaient recrutés parmi les membres de l'organisme communautaire Soutien Jeunesse, situé au nord de Montréal, et qui étaient inscrits au programme de Formation à l'intégration sociale (FIS) du Service d'éducation aux adultes de la commission scolaire couvrant ce secteur de la ville.

Le programme FIS faisait alors l'objet d'une expérimentation auprès de ces personnes aux besoins de formation particuliers. Étant en processus 
de réinsertion sociale, elles avaient besoin, par exemple, d'apprendre ou de réapprendre la gestion de leur stress ou certaines habiletés de communication. Le programme pouvait également comporter des apprentissages à l'hygiène corporelle de base, des ateliers de gestion de budget (faire son épicerie) ou d'alphabétisation, par exemple. Ainsi, par la préparation et la diffusion des émissions de radio, les participants pouvaient acquérir des techniques de recherche documentaire, de rédaction et se donner une discipline de travail. L'occasion leur était aussi offerte de structurer une présentation et par extension leur mode de penser. Le plus important était cependant lié au fait d'anticiper un passage au micro et ainsi de reconnaître une situation stressante, donc d'y faire face comme le font la plupart des gens qui sont actifs sur le marché du travail. Chacune des émissions permettait aussi à d'autres membres de la ressource d'entraide communautaire de voir présentées certaines de leurs réalisations (témoignages, poèmes, chansons, blagues, etc.), même s'ils ne participaient pas directement aux enregistrements. Tout cela favorisait la consolidation d'un sentiment d'appartenance à la ressource, en l'occurrence le centre Soutien Jeunesse, parce que ses membres-usagers étaient appelés à produire euxmêmes l'émission et à en déterminer le contenu, conformément à l'esprit du par-et-pour véhiculé par le secteur communautaire.

Pour les artisans de l'émission, il s'agissait de permettre l'expression spontanée ou préparée des personnes qui vivent des problèmes de santé mentale et de cette façon de permettre à la folie de parler pour elle-même. La tâche était parfois delicate, étant donné que les participants étaient délibérément placés dans des situations qu'auparavant ils avaient tendance à éviter parce qu'elles généraient chez eux de l'anxiété. «Les médias en général présentent le côté spectaculaire et scabreux du sujet. Folie Douce est un concept différent et novateur: en allant au fond des choses, en présentant le côté humain et en permettant à des individus vivant des difficultés de venir présenter le meilleur d'eux-mêmes, nous aidons à diminuer les tabous tout en dédramatisant.» C'est ainsi que les responsables de l'émission la décrivent sur leur site Internet ${ }^{24}$.

En plus de présenter un autre côté de la problématique, soit celui de ceux qui la vivent, l'expression à la radio se voulait l'occasion de gagner en assurance et de reprendre confiance. Toutefois, ce média a ses limites. C'est-à-dire que la radio ne permet pas que soient observées les interactions non verbales entre les participants et les animateurs. En effet, les

24. http://www.antenne.qc.ca. 
enregistrements seulement sonores entraînent une simplification considérable du matériel qui pourrait révéler, à l'analyse, une interaction basée sur une communication de type paradoxal, par exemple, lorsque le discours d'ouverture n'est pas accompagné d'une posture d'ouverture. Ce type d'enregistrements, tout comme ceux des réunions des conseil d'administration d'organismes communautaires en santé mentale, ne transmettent «guère que le contenu lexicographique et sont dépouillés d'une bonne part du matériel [permettant de qualifier une interaction] ${ }^{25} »$. Ainsi, la portée didactique d'une émission de radio est limitée par le fait que l'information livrée au public ne puisse l'être que par le son et qu'à un moment précis dans la semaine, soit lors de sa diffusion.

\section{La télévision}

La lacune inhérente au fait qu'une émission de radio ne puisse être que sonore devait être en partie comblée par la transposition de Folie Douce à la télévision. À la même époque où Folie Douce allait aussi devenir une émission de télévision, ses artisans s'associaient formellement en un organisme communautaire dont le conseil d'administration était composé majoritairement de personnes utilisatrices de services de santé mentale. Ainsi, en 1996, fut incorporé l'organisme Antenne Communications. Financé dans le cadre du programme de Soutien aux organismes communautaires (SOC) de l'Agence de santé et de services sociaux de Montréal (alors la Régie régionale), il s'agit essentiellement du regroupement des artisans de l'émission Folie Douce, qui est ainsi devenue autonome par rapport au centre Soutien Jeunesse mentionné plus haut. Antenne Communications est donc un organisme sans but lucratif qui a pour but de démystifier le très vaste sujet de la santé mentale, tout en faisant participer des personnes qui sont touchées par ce sujet à travers des activités de communication.

De janvier 1996 à mai 1997, Folie Douce fut aussi une émission de télévision présentée et coproduite par la Télévision communautaire de Vidéotron (TCV) à Montréal. Folie Douce télévision, à la différence de Folie Douce radio, fournit un matériel où l'on peut voir certaines dynamiques dont il est important de prendre conscience si l'on veut bien comprendre ce que cela peut vouloir dire que de s'être vu reconnaître un problème important de santé mentale. Martin Fortier, l'artiste-peintre

25. P. Watzlawick, op. cit. 
membre des Impatients et dont nous avons analysé précédemment quelques tableaux, est l'un des cofondateurs d'Antenne Communications. Il a plusieurs fois participé à l'émission, et nous allons maintenant revenir sur les commentaires qu'il a formulés à ces occasions.

Lors de l'émission inaugurale, Martin Fortier est accueilli par l'animateur qui lui demande d'expliquer l'une de ses œuvres où figure un asile. Le personnage va vers une «maison de créativité ${ }^{26}$ ", explique-t-il. Il s'agit d'un asile dans le sens de refuge, où il y a de multiples types d'expression: théâtre, vidéo, cinéma. Le personnage a de la difficulté à s'y rendre, «parce qu'il y a des coquelicots».

Depuis l'âge de seize ans, Martin Fortier souffre de ce qu'il nomme ses «difficultés émotionnelles». Il évoque lui-même la communication paradoxale comme lui étant insoutenable. Racontant son expérience sur le marché du travail, il dit alors: «j'ai trop de monde [...] qui me donnent des ordres, puis je ne sais plus où aller, $\mathrm{j}$ 'ai des directives contradictoires ${ }^{27}$ ».

Plus tard dans la saison, lorsque l'animateur lui demande ce qu'il pense de sa famille, Martin Fortier répond:

Je suis le bouc émissaire de la famille. On me surprotège, puis on m'empêche de respirer. Il faut toujours que je sois bien rasé, bien lavé, bien coiffé, il faut que je sois bien vêtu. Lorsque je suis bien vêtu, on me dit que je devrais porter des jeans. Ma mère me dit de me servir si je veux du lait, mon père me dit: $«[\ldots]$ assis-toi, on va te servir ${ }^{28}$.

On voit clairement ici deux éléments cohabiter, soit la situation de bouc émissaire et un environnement caractérisé par la communication paradoxale.

La neuvième émission télévisée de Folie Douce fut l'occasion d'une expérience. L'animateur a changé de place avec Martin Fortier qui s'est improvisé coanimateur. Pendant la pause, entre le premier et le second tiers de l'émission, il est passé du siège habituel des invités à celui de l'animateur et inversement. L'équipe technique n'y avait pas été préparée, parce qu'un tel changement ne pouvait avoir été convenu d'avance. Pour respecter l'effusion d'originalité, il ne fallait pas que tout ait déjà été décidé. Le défi était de permettre l'expression, la plus chaotique soit-elle,

26. M. Fortier, op. cit.

27. Id.

28. Id. 
tout en l'organisant dans le format d'une émission de télévision. L'occasion devait être saisie parce qu'il ne fallait pas présumer de la volonté future de Martin Fortier de se prêter à cette permutation. Ce ne fut que le matin de l'enregistrement qu'il exprima son désir de poser quelques questions à l'invité, un art-thérapeute. Ils allaient parler ensemble d'un symposium à venir, au cours duquel des œuvres réalisées dans des ateliers d'art-thérapie allaient être exposées. Une émission spéciale de Folie Douce couvrirait cet événement, et c'est lors de cette émission que furent recueillis plusieurs des commentaires qu'a fournis Martin Fortier à propos de certaines de ses réalisations alors exposées, et qui ont été repris dans la section précédente.

L'expérience à la radio nous indiquait que, parfois, pour une personne sensible au stress, savoir trop longtemps d'avance qu'elle devra parler publiquement peut générer une anxiété telle que l'activité sera annulée. De plus, à la radio, l'encadrement est moins rigide et moins impressionnant qu'à la télévision, ce qui favorise davantage la spontanéité. On suppose que le fait de se savoir écouté par un certain public confère une valeur à l'expérience en termes d'habiletés sociales et de communication, mais l'apparition à la télévision fait qu'on risque d'être reconnu. Il s'agit d'une étape supplémentaire dans l'acceptation de sa maladie.

$\mathrm{Au}$ cours de l'avant-dernière émission de la première saison (janviermai 1996), en guise de bilan, il y eut retour sur la saison et sur la démarche. Au début, l'une des participantes, Céline Rioux, était trop timide pour s'exprimer à la radio, ni en direct, ni en pré-enregistrement, mentionne-t-on. Sa collaboration s'est faite ainsi: l'équipe de Folie Douce téléphonait chez elle pour retransmettre ce qu'elle avait laissé sur son répondeur automatique. Ensuite, elle a pu parler «en direct» au téléphone, puis elle a franchi les étapes du processus pour finir par s'exprimer assez librement à la télévision. Quant à Martin Fortier, il répète que Céline Rioux est une artiste créative et qu'elle est pour lui une confidente. À son propre sujet, il se constate, en revoyant certains extraits d'émission, «un peu médicamenté, un peu sur-médicamenté, mon élocution... J'aimerais avoir une meilleure image à présenter aux gens qu'une personne qui a des difficultés à s'exprimer ${ }^{29}$.» À un certain moment, soit lorsqu'une entrevue avec un professeur est réalisée à l'université, on peut le voir passer en arrière-plan. Sa dyskinésie tardive, probablement conséquente à l'usage de

29. Id. 
neuroleptiques, est alors flagrante, puisque son tronc est complètement désaxé.

On peut donc dire que, afin de faire comprendre au public les multiples facettes de la problématique de la maladie mentale et de la psychiatrisation du vécu, le recours à l'image télévisée permet de voir le phénomène à l'œuvre, les effets de la dyskinésie tardive par exemple, et de mieux en comprendre les conséquences. Il s'agit donc d'un bon véhicule promotionnel, mais en principe, une émission de télévision, comme une émission de radio, ne passe qu'une seule fois. L'accès à son contenu est limité dans le temps. De plus, l'enregistrement télévisuel est d'une lourdeur assez intimidante, faisant en sorte que l'expression spontanée, voire débridée des personnes éprouvant des lacunes communicationnelles, n'est pas vraiment la bienvenue et ce, malgré l'objectif de départ qui consistait à faire connaître cette problématique en faisant s'exprimer à son sujet des personnes qui la vivent.

\section{Internet}

Nous allons maintenant proposer une technique d'intervention qui se veut conséquente aux commentaires précédents. Elle se situe à deux niveaux: individuel et collectif. Sur le plan individuel, elle prend la forme de la participation à une mesure appelée Insertion sociale (INSO, ancien programme d'expérience de travail, EXTRA). Sur le plan collectif, Internet apparaît comme un excellent moyen de diffuser de l'information et de sensibilisation, comme nous le verrons plus loin.

Telle que mise en œuvre au Centre de jour et de soir de Maison L'Échelon $^{30}$, dans l'est de la ville de Montréal, la mesure INSO était associée au programme FIS de la commission scolaire. Le programme FIS, tel qu'il était appliqué à Maison L'Échelon, était unique en son genre, du moins au sein de la commission scolaire concernée. Il reposait en effet sur l'acquisition de techniques concrètes de programmation et de communication multimédia, soit sur les TIC. Ce secteur a été ciblé pour diverses raisons, notamment parce qu'il est populaire auprès des jeunes.

Mentionnons qu'avant d'avoir pris cette direction vers les TIC, c'était dans le cadre de la production d'un journal, Le Ruisseau, que le programme FIS s'ancrait à Maison L'Échelon. Les chroniqueurs du Ruisseau y présentaient diverses chroniques, dont l'une des plus populaires était

30. http://www.maisonechelon.ca. 
celle des témoignages, souvent très émouvants, qu'ils rédigeaient concernant leur vécu psychiatrique. Ensuite, ces chroniqueurs ont été approchés par l'équipe de Folie Douce radio afin que l'émission soit alimentée de diverses manières. Par exemple, quelques témoignages ont fait l'objet de narrations à la radio, soutenues par des trames sonores parfois exécutées par d'autres chroniqueurs possédant certains talents musicaux.

Avec l'application du nouveau programme utilisant les TIC, les chroniqueurs du Ruisseau (qui a alors changé de nom pour devenir la revue Mentalité et le site webdomadaire.net) étaient notamment invités à développer leur curriculum vitæ interactif. Différents ateliers ont été mis sur pied à cette fin: traitement de l'image avec une formation sur le logiciel PhotoShop (atelier de dessin informatique), traitement de texte avec Word (atelier d'apprentissage de l'ordinateur), recherche sur Internet avec Internet Explorer, enregistrement et traitement d'éléments sonores avec WaveLab (atelier de musicothérapie). De sorte qu'en plus des compétences accumulées en matière de recherche documentaire et d'habiletés de rédaction, les chroniqueurs pouvaient aussi dresser la liste d'un certain nombre de compétences techniques associées à l'utilisation d'autant de logiciels dont ils pouvaient faire état dans leur c.v., conformément à la situation du marché du travail où l'usage de l'ordinateur s'est généralisé.

La création d'un site Web ou d'un montage multimédia requiert en effet un nombre assez important d'étapes, ce qui fait que ce n'est pas à la portée de tout le monde. Aussi, le site créé par la clientèle est accessible de partout, à tout moment. De sorte que lorsqu'un chroniqueur est en visite dans sa parenté à l'occasion de Noël par exemple, il peut montrer son c.v. interactif à un cousin, à un oncle, etc. Certains ont ainsi pu susciter de l'admiration chez leurs proches qui, auparavant, étaient plutôt insensibles à d'autres types de réalisations: bricolage, peinture, macramé, etc. L'entourage immédiat change alors d'attitude. Étant souvent incapable de réaliser des pages Web, plusieurs sont impressionnés, alors qu'auparavant ils trouvaient puériles les activités auxquelles se livrait un proche victime de la maladie. Finalement, le prestige du secteur compense un peu pour la faible estime de soi et rejaillit partiellement sur leurs artisans, favorisant un certain rééquilibre.

De plus, les ateliers mentionnés n'étaient pas seulement réservés aux chroniqueurs du Ruisseau, puisqu'avec le temps, ils ont été intégrés à la programmation saisonnière du Centre de jour et offerts à l'ensemble de ses quelque 400 membres. Par exemple, la café-rencontre du mercredi soir, 
fort populaire, a un peu pris la forme d'un café-Internet, parce qu'en l'absence des chroniqueurs du journal, quelques participants pouvaient accéder aux ordinateurs pour y surfer sur Internet.

En tant que composition multimédia, par définition la mise en ligne d'une page Web se déploie à plus d'un niveau, comportant des éléments textuels, visuels ou sonores. Ces trois formats de communication peuvent être intégrés de manière originale, voire paradoxale. Or, comme nous l'avons mentionné, la communication paradoxale a souvent été l'instrument de l'exclusion initiale. Il s'agit donc de se la réapproprier, mais cette fois avantageusement. En effet, une page Web est essentiellement composée d'une série de « 0 » et de « $1 »$. Pour décrypter un sens à cette série, l'ordinateur se fera indiquer où commence la séquence et où se trouvent les parenthèses (balises html) qui font que cette série constitue une équation qu'il devient possible de recomposer. C'est donc le contrôle sur cette ponctuation que se trouve à exercer le programmeur de la page en question.

Tel que nous l'avons mentionné plus haut, dans le cadre d'une communication paradoxale, la manipulation est entretenue grâce à l'emploi du double langage paradoxal, lequel consiste en un décalage plus ou moins marqué entre ce qui est dit et ce qui est fait. C'est comme si, dans un film, on faisait entendre le vrombissement d'un réacteur de jet tout en projetant des images de voilier. Plus le décalage ou l'anachronisme est large, plus la communication est paradoxale. Plus le décalage est grand entre ce qui est dit verbalement et ce qui est dit corporellement ou ce qui est fait, plus grand est le sentiment de trahison. Plus grande est l'amertume face à ce qui est perçu comme de l'hypocrisie. D'où la volonté de retrait de ce jeu cruel. À celui-ci, quoi que l'on dise ou que l'on fasse, on perd à tout coup, peu importe le choix des options, le scénario a toujours la même fin. L'exclusion étouffe la parole de la folie sur elle-même et l'empêche de se déployer stratégiquement, de se libérer de la communication paradoxale imposée. Il s'agit, en quelque sorte, d'une réalité virtuelle multivoque, à laquelle sont confrontées certaines personnes qui en deviennent finalement des expertes, malgré elles.

«Le virtuel nous fait accéder à un monde possible, à une possibilité du monde que l'on se construit ${ }^{31}$.» En effet, composer sa page Web est l'occasion de se projeter différemment. La possibilité est offerte de recomposer sa personnalité, d'avoir le contrôle sur cette composition, libre des stig-

31. M. Wawrzyniak, «La virtualité à l'adolescence. De la virtualité à la déréalité», L'information psychiatrique, vol. 74, janvier 1998, p. 19-26. 
mates physiques ou psychologiques de la maladie. Et il va sans dire qu'une fois familiarisés avec les techniques de composition de pages Web, les participants sont devenus des habitués des TIC, pouvant surfer à volonté sur la grande toile. Il faut toutefois avoir à l'esprit que le virtuel, jusqu'à un certain point, peut ébranler le sentiment de la réalité et conduire à l'irréalité psychotique. Nous dirons de cette irréalité qu'elle est grisante. C'est pourquoi elle séduit en particulier l'adolescence, ce moment décisif de l'organisation structurale de la personne. L'adolescent pourra alors s'enfermer dans le virtuel, ce qui, entre autres, a pour «conséquence de freiner chez les jeunes les pouvoirs de réalisation ${ }^{32}$ ». Jouer permet de mieux supporter la réalité, en la narguant, et c'est ce qui explique que les adolescents soient particulièrement friands de jeux vidéo, laissant libre cours à leur attrait pour la pré-intégration psychologique. Or si faire une incursion hors de soi peut être une expérience divertissante, le danger est cependant réel de devenir en quelque sorte dépendant de cette griserie. En effet, «les régressions psychotiques se manifestent plus aisément lorsqu'il existe une faible capacité intégrative du Moi, [...] où de sévères difficultés développementales à la phase séparation-individuation sont observées ${ }^{33}$ »).

Avec les précédentes réserves en tête, on voit à quel point l'expression sur Internet, grâce à la mise en ligne de sa page Web, permet de surmonter les limites associées aux autres possibilités d'exercice de la communication dont nous avons esquissé les principaux enjeux. De plus, les avantages de ces autres modes d'expression peuvent être cumulés sur une page Web, parce qu'on peut y retrouver à la fois des images (comme les tableaux de Martin Fortier), des textes (comme les témoignages des chroniqueurs du Ruisseau) et du son (comme la musique produite dans le cadre d'un atelier de musicothérapie). On peut aussi y trouver des extraits vidéo et des hyperliens conduisant à une grande variété des sites proposant de l'information diversifiée et accessible en tout temps, et on peut échanger avec des interlocuteurs de toute provenance sans être vu et nécessairement perçu comme quelqu'un qui a des problèmes de santé mentale. On peut alors se présenter sous un autre jour et ainsi goûter à la liberté de décider comment se projeter en étant l'instigateur, plutôt que la victime, de la communication paradoxale se déployant à plusieurs niveaux. Une sorte d'hypnose de l'écran peut ainsi se révéler des plus captivantes. Inhibant en même temps l'intellect typiquement rationnel, elle peut favoriser chez

32 Ibid., p. 20.

33. P. Lalonde et al., Psychiatrie clinique: une approche bio-psycho-sociale, seconde édition, t. 1, Boucherville, Gaëtan Morin éditeur, 1999. 
plusieurs l'expérience positive compensatoire de se savoir l'artisan de cette hypnose captivante précisément grâce à leur connaissance intime de la communication paradoxale. Ainsi: «À l'inverse du monde réel, le monde virtuel ne résiste pas à nos désirs [et] donne un sentiment de pouvoir magique, hautement gratifiant par rapport au sentiment chronique de notre manque d'être, de notre inachèvement et de notre soumission à toutes les frustrations de la vraie $v^{34}{ }^{34} . »$

Il s'agit en quelque sorte de combattre le feu par le feu, soit de mettre à profit l'irrationalisme caractéristique de la psychose dans un cadre qui correspond justement à l'ère post-rationaliste qui s'annonce avec les technologies numériques. Ces dernières, selon Hervé Fischer, changent en particulier le rapport à la connaissance et à l'enseignement, lequel devra renoncer à une partie de son autorité. L'enseignement devra être beaucoup plus souple, plus convivial. Par exemple, avec la lecture kangourou, un élève peut suivre le chemin qu'il veut parce que l'arborescence d'un cours à distance sur Internet lui permet de commencer sa lecture, de la terminer puis de la reprendre où il veut, dans l'ordre ou le désordre que lui inspire sa curiosité. Le cheminement didactique imposé et linéaire, hérité de l'âge de l'imprimerie, risque de faire fuir les clientèles estudiantines de demain. L'enseignement académique devra en tenir compte et autant que possible se donner de manière interactive, à l'image des technologies postrationalistes, au risque de voir une clientèle déjà démographiquement de moins en moins nombreuse lui tourner le dos.

\section{Conclusion}

Avec la prolifération des médias interactifs, «c'est à une entrée en force du monde virtuel que nous assistons, un monde avec ses effets de fascination, voire de captivation ${ }^{35}$ ». Bien que le risque soit réel pour certains de se retrouver démunis face à ce terrible désarroi qu'est la vie adulte, l'exercice de la virtualité et celui de l'imaginaire, chez d'autres, «jouent un rôle organisateur d'expression et d'élaboration, ces recours les aidant à mieux réaliser le réel, c'est-à-dire à en faire plus facilement leur réalité $^{36} \gg$.

34. H. Fischer, CyberProméthée, l'instinct de puissance à l'âge du numérique, Montréal, VLB éditeur, 2003.

35. M. Wawrzyniak, op. cit.

36. Ibid., p. 22. 
Toutes les considérations ci-haut évoquées nous ont donc conduits à cibler un secteur particulier à exploiter, soit le domaine du multimédia interactif. D'une part, lorsqu' on travaille pour un site Internet, par exemple, on peut le faire de n'importe où, là où il y a un ordinateur et une ligne téléphonique, de la maison notamment. Le télétravail permet à ceux qui y sont sensibles d'éviter le stress des contacts sociaux en faisant tout de même activement partie d'une collectivité. D'autre part, ce champ d'activités est encore en pleine croissance et beaucoup de prestige y est associé, alors que, comme la littérature le démontre abondamment, la maladie mentale génère souvent un grave manque d'estime personnelle (ou est-ce l'inverse?).

L'Internet s'annonce donc comme l'espace symbolique de l'ouverture infinie et de l'investissement psychique libertaire. En outre, la communication par Internet permet au libre-penseur ou au simple citoyen de communiquer sans la médiation d'un éditeur, et ainsi de contourner l'ordre raréfiant des discours, lequel invalidait de facto la subjectivité d'un patient en ne la rendant pas disponible. "Quand j'y navigue, en voyageur solitaire, protégé par mon intimité, libre de mes pensées, de mes émotions, de mes aveux, je sais aussi que j'évolue sous le regard des étoiles (ou de Dieu) ${ }^{37} . »$ C'est ainsi qu'on peut y recouvrer la valeur d'être qui est si souvent refusée dans le cadre de l'interaction psychiatrique. De sorte qu'Internet pourrait offrir une excellente méthode thérapeutique, précisément parce qu'il permet d'échapper aux contraintes et frustrations du monde réel.

Et pour ceux qui sont enfermés, reclus, [Internet] représente le vitrail qui ouvre sur un ailleurs, là où le malade mental retrouvera ce qui lui a manqué et a souvent causé son trouble: une communication fusionnelle avec une communauté virtuelle dans laquelle il s'intègre symboliquement, l'accès à un être supérieur, un monde irréel, fluide, sans gravité terrestre, où l'âme vole en apesanteur, où les difficultés du principe de réalité s'évaporent, un monde gratifiant, énergique, lumineux, interactif, qui obéit au clic magique et en même temps réserve les surprises excitantes d'une navigation en arabesque, donc un monde qui évoque sous beaucoup d'aspects un meilleur transcendantal infiniment plus

37. H. Fischer, «Préface», dans J.-F. Pelletier, Réclusion et Internet, Québec, Presses de l'Université du Québec, collection «Problèmes sociaux et intervention sociale», 2005. 
attrayant que l'ici-bas de la vallée de misère et des murs de l'enferment, favorisant une émotion spirituelle ${ }^{38}$.

Dans Le choc du numérique ${ }^{39}$, Hervé Fisher dégageait ce qu'il appelle une loi paradoxale, à savoir que, malgré sa nature technologique, Internet agit comme un psychotrope et active l'inconscient. "Le réel s'y reflète, certes, et même y fait sans cesse des incursions, mais comme les vagues sur la plage, sans la perturber. Ici, c'est l'imaginaire symbolique qui a force ontologique et $s$ 'impose. Le réel ne fait qu'y affleurer ${ }^{40} »$, écrivait-il encore en 2005.

Sur Internet, on peut même y changer d'identité, et donc s'affranchir au moins un court instant de celle d'un malade mental. On peut notamment développer des liens sociaux, qui pour être virtuels n'en sont pas moins réels, en se débarrassant des infériorités civiles qui sont prétextes à l'exclusion sociale. C'est pourquoi, selon nous, son potentiel thérapeutique et de revalorisation devrait faire l'objet de recherches plus approfondies.

Pour terminer, invitation est lancée au lecteur de visiter les réalisations multimédias qui se trouvent à l'adresse suivante: www.polaristo.com/jfpelletier/cahiersderecherchesociologique. Il pourra notamment voir les peintures de Martin Fortier et accéder aux témoignages des chroniqueurs du Ruisseau, ainsi qu'à un feuilleton multimédia ayant jeté les bases de la programmation informatique du Centre de jour de Maison L'Échelon (ateliers de dessin, de production sonore et musicale et de rédaction). Toutes ces communications sont du domaine public, elles prennent même le public à témoin pour en faire le tiers unificateur.

Jean-François PELLETIER

$\mathrm{PhD}$, coordonnateur de recherche à la direction des

Technologies de l'information et de la Recherche des centres de réadaptation Lisette-Dupra et de l'Ouest de Montréal

38. Id.

39. H. Fischer, Le choc du numérique, le triomphe des cyberprimitifs, Montréal, VLB éditeur, 2001.

40. H. Fischer, «Préface», op. cit. 
Pensée psychotique, technologies des communications et réadaptation psychosociale 277

\section{Résumé}

Cet article propose une réactualisation de la théorie de la communication paradoxale (école de Palo Alto) à la lumière des technologies de l'information et des communications, très en vogue de nos jours. Après avoir campé les grands enjeux théoriques de cette approche antipsychiatrique, cet exposé revient sur diverses modalités d'exercice de la communication comme moyen de favoriser la réadaptation psychosociale des personnes ayant, ou ayant eu des problèmes de santé mentale. Internet émerge alors comme un secteur à explorer et à développer. D'une part, son caractère multimédia en fait un mode de communication typiquement paradoxal. D'autre part, il fait en sorte que soient cumulés les avantages des diverses techniques étudiées (art-thérapie, écriture journalistique, radio et télévision), ce qui facilite l'expression d'un discours affranchi de l'ordre raréfiant du discours institutionnel. Ce dernier dénigre en effet la parole plus ou moins chaotique du patient en en faisant le symptôme d'une maladie à juguler. C'est ainsi que, grâce au potentiel thérapeutique d'Internet, la pensée psychotique peut recouvrer une certaine valeur et c'est ainsi que ses porteurs peuvent communiquer avec autrui, c'est-à-dire directement avec la société et aussi avec un certain au-delà transcendantal.

Mots clés: théorie de la communication, anti-psychiatrie, technologies de l'information et des communications, problèmes de santé mentale, virtualité

\section{Abstract}

This article intends to revisit the paradoxical communication theory (Palo Alto) in the light of information and communication technologies, which are now very popular. After addressing the main theoretical implications of this anti-psychiatric theory, this article evaluates different modalities employed to facilitate the psychosocial rehabilitation of persons who have, or had, mental health problems. The Internet emerges as a domain to explore and develop. On one hand, its multimedial character makes it a typically paradoxical form of communication. On the other hand, it allows the advantages associated with other communication techniques (art-therapy, journalistic writing, radio and television) to be cumulated in order to facilitate the expression of a discourse free of the rarefying order of institutional discourses. The latter denigrates the more or 
less chaotic word of the patient, making it the symptom of a disease to halt. This is how, with the therapeutic potential of the Internet, psychotic thinking can recover a certain value, its carriers being able to communicate with others, that is, directly with society and also with a kind of a transcendental path.

Key words: communication theory, anti-psychiatry, Information and Communication Technologies, mental health problems, virtuality

\section{Resumen}

Este artículo propone reactualizar la teoría de la comunicación paradójica (escuela de Palo Alto) a la luz de las tecnologías de la información y las comunicaciones, muy de moda en éstos días. Luego de una descripción a propósito de los grandes desafíos teóricos que están en juego en éste enfoque anti-psiquiátrico, nuestra exposición regresa hacia las distintas modalidades de ejercicio de la comunicación como medio facilitador de la readaptación psicosocial de las personas que sufren o han sufrido problemas de salud mental. Internet surge entonces como un sector que debe explorarse y desarrollarse. Por una parte, su carácter multimedia lo transforma en modo de comunicación típicamente paradójico. Por otra parte, al permitir acumular las ventajas de las distintas técnicas estudiadas (arte-terapia, escritura periodística, radio y televisión), facilita la expresión de un discurso liberado del orden poco común del discurso institucional. Efectivamente, éste último menosprecia la palabra más o menos caótica del paciente convirtiéndola en el síntoma de una enfermedad que debe yugularse. Por ello, gracias al potencial terapéutico de Internet, el pensamiento psicótico puede recobrar un determinado valor permitiendo que sus portavoces pueden comunicar con otros, es decir, directamente con la sociedad y también con un determinado más allá trascendental.

Palabras clave: teoría de la comunicación, antipsiquiatría, technologías de la informacíon y de las comunicaciones, problemas de salud mental, virtualidad 